\title{
Redes y conjuntos de acción: para aplicaciones estratégicas en los tiempos de la complejidad social
}

Tomás R. Villasante - Universidad Complutense de Madrid ${ }^{1}$ Pedro Martín Gutiérrez - Universidad de Valencia

\begin{abstract}
Resumen
En este trabajo se propone una metodología participativa y reflexiva de desarrollo de mapas sociales. Se apuesta por incorporar en el sociograma, de modo flexible, el grado de afinidad de las relaciones junto a otros ejes sociales e ideológicos. Se presenta el concepto de conjuntos de acción, y se discuten las implicaciones de este enfoque, tanto en lo que hace referencia al muestreo como a aspectos teóricos más generales.
\end{abstract}

Palabras clave: Sociogramas - Conjuntos de acción - Relaciones de afinidad Muestreo.

\begin{abstract}
In this paper we propose a participative and reflexive approach to social maps. Several social and ideological categories may be applied in the design of sociograms. The concept of "action set" is presented, as well as its consequences in theoretical terms and for sampling purposes.
\end{abstract}

Key words: Sociograms - Action set - Affinity relationships - Sampling.

\section{Los retos actuales de la teoría de redes}

Hace años mantuvimos en la Facultad de Ciencias Políticas y Sociología, de la Universidad Complutense de Madrid, un debate que organizó Narciso Pizarro con una serie de profesores norteamericanos sobre la actualidad del Análisis de Redes². Se recordaron en este coloquio las fases previas: su comenzar con la antropología inglesa, la escuela de Manchester, su uso para situaciones complejas donde se mezclaban usos tradicionales africanos o asiáticos con nuevas costumbres occidentales, la formalización en "grafos", etcétera. Esto representaba en los años 50/60 todo un avance, pues no sólo mostraba la potencialidad de una técnica descriptiva, sino que nos colocaba ante las posibilidades de un pensamiento que implícitamente apuntaba a la complejidad de las relaciones humanas graficadas desde los vínculos de lo cotidiano.

\footnotetext{
${ }^{1}$ Enviar correspondencia a: invpar@cps.ucm.es. Queremos agradecer a los seminarios CIMAS, donde hemos presentado este trabajo, los debates y sugerencias que nos han hecho (especialmente a Lucrecia Olivari, Javier Garrido y Manuel Montañés).

${ }^{2}$ Vid. VV.AA. (2000).
} 
Creemos que quienes más han aprovechado esta línea han sido los profesionales de la psicología social. En el cono sur, desde la llegada de Pichón-Rivière y la formulación de la "teoría del vínculo" (1991) hasta los trabajos más recientes compilados por Dabas (1993) se han desarrollado, con gran eficacia en los tratamientos grupales, estos enfoques que inciden en que los problemas no son tanto de las personas en sí sino de las redes que los habitan. $Y$ en consecuencia, que al abordar las redes sociales que nos constituyen es la mejor forma de producir los cambios que en cada caso sean pertinentes. Quizás un primer reto está en ver si es posible pasar a ámbitos de mayor envergadura, comunidades territoriales, estructuras de poder en la sociedad, etcétera. La economía, la sociología, la política, tendrían aquí mucho que avanzar.

Hay estudios interesantes sobre cómo se interrelacionan entre sí las elites económicas y políticas, y también cómo los pobres de las comunidades marginales logran sobrevivir, u organizar sus movimientos sociales (Lomnitz 1977). En Canadá se ha ido formando una escuela que ha potenciado estos estudios y ha significado un avance importante en los años 80 y 90 (ver B. Wellman, etcétera). Pero a partir de aquí se ha intentado una formulación en términos de matrices numéricas, intentando una mayor precisión en las descripciones de los vínculos y relaciones, que más que ayudar a superar los retos creemos que han dificultado la comprensión de la complejidad de estos procesos. Desde luego la formulación en teoría de grafos y en matrices matemáticas puede ser útil y mostrar ciertas precisiones (Ver White, etcétera). Pero nos puede despistar también de lo más profundo de estas líneas de investigación.

Por ejemplo los artículos de Granovetter (2000:41-56) sobre la "fuerza de las relaciones débiles" aportan más a las ciencias sociales que tantas otras descripciones de relaciones de casos concretos, muy precisas numéricamente pero con poca profundidad en situaciones complejas. El que un grupo o una persona (por ejemplo, puede ser el caso de un técnico de una institución) que llega de fuera y establece un puente entre varias redes, precisamente porque no es conocido y pueda elegir dónde situarse, tenga una "fuerza" por esa unión y no por ser conocido en ese lugar, es algo que afecta a las relaciones de poder y que puede implicar cambios de estrategias locales. Es decir, nos estamos planteando cuestiones de fondo, como son: la cuestión de la complejidad del poder, la cuestión de la temporalidad de los procesos y sus estrategias, la cuestión de las relaciones entre los ámbitos macro y microsociales. 
Por esto hemos reclamado (Villasante, 2000) una nueva fase dentro de los estudios de redes. Unos estudios que se preocupen por estas cuestiones a partir de la complejidad de los "datos", que no pueden ser tabulados en 0 ó 1 simplemente, se da o no se da la relación. Sino que cada vínculo tiene en sí mismo un carácter más de "constructo" (temporal y local) que de "dato en sí" (a fotografiar); tienen también caracteres múltiples, al ser reflejo de estructuras económico-ecológicas tanto como de luchas de ideologías o como sentimientos emocionales; y además hay influencias de la vida cotidiana más local tanto como lo que le llega de la globalidad en la que vivimos. Desde los años 80 , pero sobre todo en los 90 , hemos aportado una serie de estudios concretos en esta dirección, que nos vienen dando algunos resultados para la elaboración de diagnósticos y planificaciones estratégicas, con utilidades en diversos ámbitos.

Las metodologías participativas que hemos venido usando nos parece que dan más juego que las de tipo cualitativo que usaban los antropólogos ingleses, o las cuantitativas que han venido usando los sociólogos norteamericanos. No se trata de un enfrentamiento entre diversos tipos de técnicas, pues todas las usamos, sino de cómo caracterizar los vínculos que estudiamos y que son base para los cambios que se pueden abrir. Si la unidad de estudio son los vínculos más que sus soportes, y sus características los hacen tan complejos y cambiantes, tan paradójicos a veces, fruto de construcciones colectivas, tendremos que usar prioritariamente metodologías con capacidad de re-construcción plural y procesual. Metodologías donde los propios soportes (grupales y personales) de los vínculos puedan pasar de ser analizados a ser analizadores, a intervenir con sus actos y posiciones en la complejidad de los procesos.

\section{Los usos del socio-grama y sus sentidos ${ }^{3}$}

El psico y el socio-grama se han usado mucho en psicología social, y también se han ido extendiendo a otras disciplinas, como la pedagogía, la comunicación, la sociología, etcétera. Casi siempre han mantenido la característica de construirse a partir de alguna persona, grupo o referente central (lo que se suele llamar "egocéntrico"). Es decir, cómo puede conseguir los votos el candidato del Partido del Congreso en la India, o el del PRI en México, a partir de las relaciones que tal candidato establece con las diferentes redes locales en cada caso, son dos ejemplos clásicos. A veces esto se ha hecho con los Diagramas de Venn, y a veces con otras formulaciones gráficas, pero en general sin incorporar otras variables que no sean el tipo de relaciones por sí mismas. Con ello se le otorga a este tipo de esquemas

\footnotetext{
3 La realización práctica del socio-grama en un proceso participativo ya ha sido expuesta en otros trabajos: vid. Martí, 2000; Villasante, 2002, Martín, 1999; Martín, 2001.
} 
gráficos y las relaciones que contienen un valor explicativo por encima de otras variables que no aparecen reflejadas en tales cuadros. Es decir tenderíamos a simplificar la explicación a una sola variable.

También tendemos a medir todo el conocimiento de las redes en grado de lejanía o cercanía al sujeto o grupo de referencia. Y eso no nos permite ver con claridad lo que no conocemos, simplemente porque no lo podemos apreciar desde donde estamos. Esto podría ser más justificable en psicología toda vez que las influencias para el cambio dependen de la conciencia que se tenga de los vínculos, pero en cuanto se llega a ámbitos mayores las cosas no dependen tan sólo de lo que uno conoce. Cambiar el foco para ver desde tres o más variables una misma situación nos puede ayudar a complejizar el panorama, y no hacerlo desde un sujeto central sino desde un tema concreto, donde el sujeto investigador se pueda ubicar cuando ya estén situados los demás grupos y sectores y las relaciones básicas establecidas, nos parece que es un avance en estas metodologías.

No siempre se hacen los mapas sociales o los socio-gramas desde la posición de un referente grupal o personal, a veces es un referente temático. Pero aun siendo un tema concreto la forma de establecer las relaciones suele ser a partir de las personas y los grupos que más creemos conocer. $Y$ esto suele llevar a colocar en el centro de las relaciones, gráficamente, y a contabilizar, numéricamente, más a los afines que a los ajenos o a los antagónicos ${ }^{4}$. Se crea así una imagen distorsionada de la realidad, sin darnos cuenta de lo periféricos que podemos ser de un proceso nosotros y nuestros afines. Observar al observador, ser auto-críticos de estas observaciones es fundamental para la auto-reflexividad compleja que pretendemos de unas metodologías que trabajan con vínculos que siempre están en proceso y que tienen muchos efectos paradójicos.

Antes de colocar las instituciones, grupos, sectores, y sus relaciones, previamente colocamos dos ejes para enmarcar con dos pinzas o focos más de contraste. Con un eje, pinza o foco, tratamos de capturar o iluminar una serie de situaciones estructurales, también construidas, pero con más profundidad histórica, heredadas, y que nos dan el contexto de los condicionantes más macro (como la clase social, el marco ecológico, etcétera). Es interesante no olvidarse de estos elementos "en si" de la situación, porque muchas de las cuestiones del poder no se juegan tan sólo en la vida cotidiana, sino que vienen marcadas por algunos elementos "objetivables"

\footnotetext{
${ }^{4}$ Nos referimos a estas posiciones en relación a la afinidad en las redes: el sujeto de referencia inicial es el "nosotros" que representan los afines que, por orden de proximidad, tienen un "vosotros" más cercano encarnado por los diferentes y un "vosotros ampliado" que son los ajenos. Por último "ellos" serán los más alejados de nuestras propuestas y con los que, como luego veremos, las relaciones suelen ser de conflicto.
} 
(y aquí se abre otra discusión sobre cuales sean estos). Sea cual sea la posición con que se quiera medir estos condicionantes más estructurales, cabe establecer siempre un enfoque, eje, o pinza que trate de ponerlos en presencia.

El otro eje previo, pinza o enfoque, es desde las posiciones de cada agente o grupo, más estratégicas, programáticas, ideológicas, que se dan dentro de un tema y de un territorio, en un momento dado. Sobre todo en los grupos formales, las instituciones, y los sectores más significativos, sobre un tema concreto que tratemos de resolver, siempre hay posiciones tomadas con cierta claridad, al menos como eslóganes o estereotipos. Cabe diferenciar este tipo de medida o percepción de la que podríamos llamar emocional en los vínculos. Una cosa es que se pueda estar de acuerdo en una posición para hacer algo concreto y otra que se tenga confianza con ese grupo o persona. Una cosa son las relaciones de miedo o de resquemor entre sectores o instituciones, de tipo más emocional, y otra que se coincida en tal programa o acción en un momento dado. En este eje nos preocupa distinguir entre, por ejemplo, los más afines a los objetivos de lo que se quiere hacer, frente a los más antagónicos. $Y$ por medio seguro que hay posiciones con varias diferencias, o incluso ajenas a todo este tema.

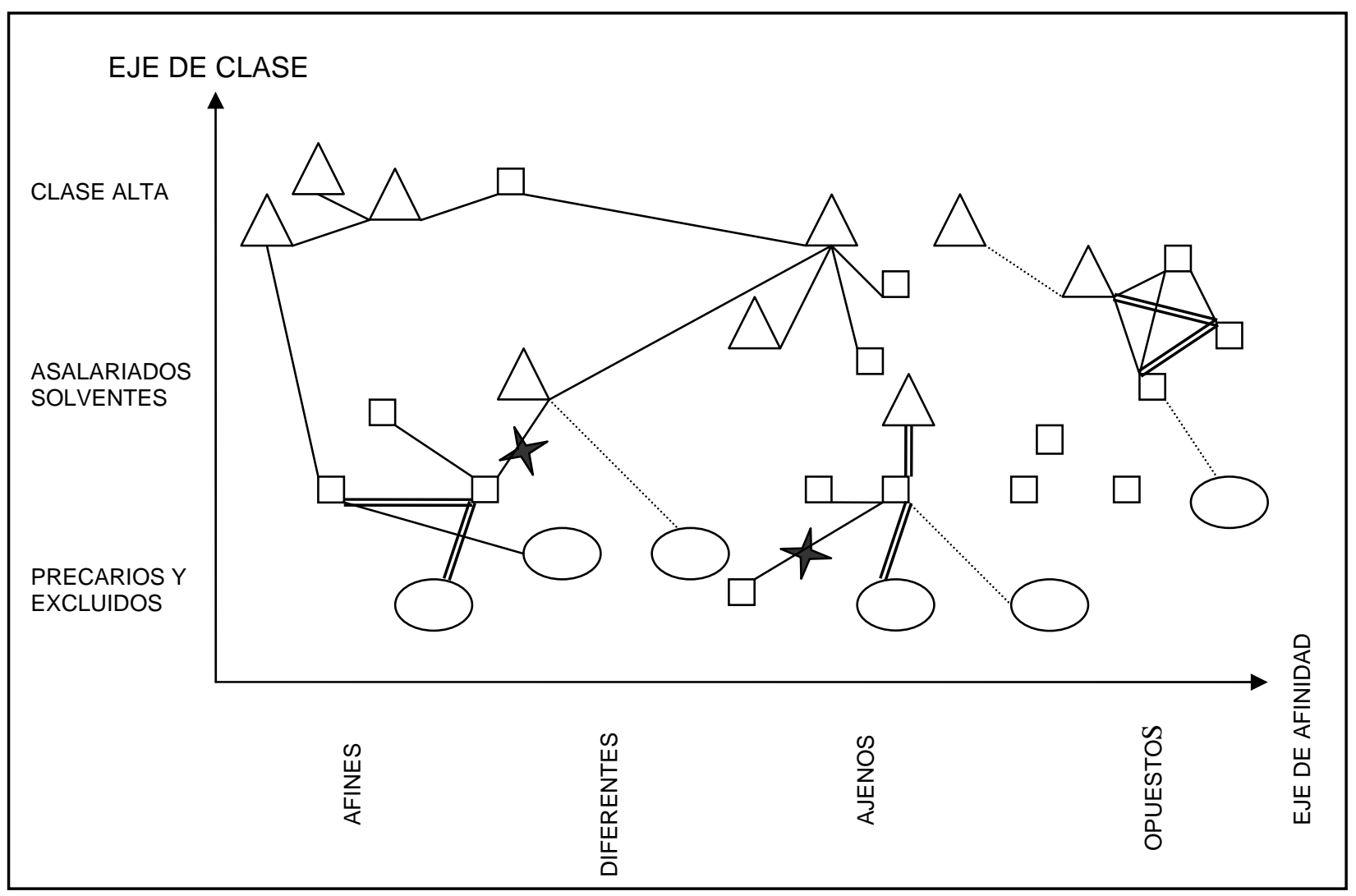

Cuadro 1. Sociograma y relaciones de afinidad

Fuente: elaboración propia 
Tendríamos así dos ejes o enfoques previos (el más estructural, y el más ideológico) con los que enfocar las relaciones emocionales, más cotidianas, que nos mostrarían los vínculos de confianzas/desconfianzas que es la tercera variable, o foco, que queremos construir también. Colocamos los grupos y sectores que son soportes de los vínculos, no en función de las relaciones sino en función de sus posiciones respecto a los condicionantes estructurales, y a sus condicionantes ideológicos. Por lo tanto no están posicionados respecto de ningún grupo de referencia, sino a un tema con características estructurales e ideológicas. Y de esta forma nos suelen aparecer cuadrantes vacíos o con grupos poco delimitados, que en un ejercicio participativo, nos permiten una reflexividad muy interesante sobre la observación de los observadores. Se formulan por los propios participantes en el diseño las dudas que de otra forma no se formularían, y esto es muy interesante para el rigor metodológico del análisis de redes.

Solemos poner distintas figuras gráficas (Triángulos, Cuadrados, Círculos) según las posiciones de partida "organizativas" de las que suponemos que parten cada grupo o sector. Por ejemplo en la vida cotidiana de un barrio o una región hay sectores, bastantes, no organizados respecto al tema que estamos tratando (Círculos), como suele suceder con pandillas, comentarios de bares o mercados, etcétera... Pero también algunos grupos con una estructura formal organizativa, que pueden influir (Cuadrados), como ONG, Asociaciones, Profesionales, etcétera. Y sin duda hay instituciones o corporaciones que están por encima de la comunidad, pero que tienen poder, al menos simbólico (TV, Iglesia, Gobernantes, etcétera), para incidir en ella (Triángulos). Podemos hacer una primera identificación inicial, participadamente, por estas características, para luego verificar más documentalmente dónde se sitúa cada cual, y que relaciones mantiene con los demás.

Nos interesan los tipos de "posiciones discursivas" que adoptan quienes se manifiestan ante el tema dado. Cuando hablamos no solo decimos cosas, sino que nos estamos diciendo a nosotros mismos, es decir, desde donde estamos hablando. Si hablamos por hablar con los tópicos o estereotipos de una conversación convencional de bar, es muy distinto de si hablamos tratando de convencer de una posición ideológica, religiosa, comercial, etcétera, o incluso cuando alguien habla por encima de las disputas, como "sentando cátedra", colocándose como una posición incuestionable. Son distintas posiciones a tener en cuenta en la comunicación, pues no sólo está el poder de lo económico y estructural, o de las posiciones ideológicas de los grupos, sino también el poder mediático de lo que se suele dar como opinión pública. $Y$ todos estos aspectos creemos que se han de 
tener en cuenta, si se está pensando en hacer operativos los objetivos que se persiguen.

En un análisis de redes acabamos colocando los vínculos, que son los elementos más importantes de todo el esquema. Y lo son porque a partir de ellos es desde donde podemos empezar a cambiar situaciones que nos preocupan. Son vínculos emocionales que resumen, en lo concreto de lo cotidiano, muchas de las condicionantes que se han ido acumulando, pero no como son simples reflejos de la estructura o de la ideología. Se puede coincidir en la clase social, e incluso ideológicamente, y sin embargo no coincidir con el estilo de hacer las cosas. Se puede coincidir en la forma de hacer las cosas (tanto si es horizontal y muy participativa, como si es vertical y paternalista) entre personas de distintas clases sociales o de distintas ideologías (por ejemplo en una fiesta o en el fútbol). Los vínculos nos dan otra dimensión posible, que no depende tanto de los condicionantes económicos o de los ideológicos, sino de la formación emocional y de las historias cotidianas vividas en las familias, entre amigos, vecinos o en el trabajo.

Por ejemplo, en un cuadrante entre los ejes ya mencionado, pueden coincidir dos grupos que se disputan ser los líderes del proceso, aunque estén de acuerdo en los mismos objetivos de mejora para la comunidad, y que respondan a las necesidades estructurales de los mismos sectores populares. $Y$ no pocas veces un proceso fracasa por esta causa de los protagonismos, de la misma manera que muchos procesos avanzan mucho mejor cuando se crea un clima de cooperación y buen ambiente, más allá de lo estructural y lo ideológico. También es interesante construir una serie de símbolos gráficos (si se quiere hacer participadamente con los propios sujetos implicados) para establecer diversos valores para los grados de confianzas o de miedos que se suscitan en cada caso. Con una pequeña gama de trazos se pueden ir colocando los vínculos de conocimiento o de distancia, de conflicto o de amistad, etcétera.

Desde luego, cuando esto se hace la primera vez con un grupo en un territorio, no deja de ser una hipótesis, más o menos validada por el conocimiento que tal grupo tenga de su realidad cercana. Pero no cabe duda que si se puede comparar con los socio-gramas de otros grupos se ganará en verosimilitud, y que de todas maneras habrá que hacer un trabajo de campo para documentar dónde se colocan cada grupo o sector a sí mismos, documentadamente. Lo que implica que cada cierto número de meses se han de seguir haciendo estas "radiografías" (cada vez menos “borrosas") de los vínculos implícitos que se generan en torno al tema, y cómo van 
cambiando. No se trata por tanto de dar una descripción de la realidad, sino la construcción en talleres sucesivos de cómo se van perfilando los oscuros, blancos y grises de cada "radiografía", según las interpretaciones del equipo "experto" (que en este caso cuenta con los grupos de la propia población tanto para dar la información base, como para contrastarla y validarla).

\section{EI socio-grama como muestra reticular tentativa}

Una muestra de las posiciones fundamentales que nos interesan conocer, en cuanto a sus motivaciones de cara a los objetivos que perseguimos, es algo muy importante para saber conjugar qué fuerzas y con qué sentidos se están construyendo. Nos interesan los motivos de los que apoyan el proyecto, tanto como los que están en contra. Tanto nos interesan los más poderosos, que cuantitativamente no suelen ser muchos, como los numerosos que se pueden ver afectados, aunque no tengan mucho poder. $Y$ tanto los grupos organizados y activistas, como los ajenos pero que en un momento se pueden poner a favor o en contra. Para un juego de estrategias hay que tener en cuenta muy diferentes posiciones, y no solo las que nos gustaría que hubiera. Como se deduce de lo dicho, no son los criterios cuantitativos los principales para seleccionar a quién debiéramos entrevistar, porque puede haber mucha gente que esté ajena al tema y que sólo va a tomar posición a partir de las propuestas que se le presenten. También nos interesa la posición de la mayoría ajena, pero primeramente tenemos que conocer las minorías que están elaborando sus propuestas y contrapropuestas.

Por esto hacer una distribución proporcionalmente homogénea por edades, género, clases sociales, y formas culturales, no es lo que interesa más para conocer las redes que hay en torno a un problema, y las bazas que se están jugando entre los implicados. Quizás para los sectores informales ajenos, sobre todo en una población grande y que no se conozca mucho, pudiera ser útil hacer alguna distribución de entrevistas de grupos que nos diera información sobre las distintas perspectivas. Pero parece más aconsejable empezar por conocer tanto las motivaciones que han convencido a los grupos más afines, seguramente para insistir sobre ellas, y también cuáles son los argumentos de los más opuestos (precisamente para poder rebatirlos o para aprender de ellos). Saber también las diferencias, de los diferentes aunque cercanos, que son con los que más se suelen pelear unos y otros dirigentes. Suele haber cuestiones de matiz que se agrandan por motivos no siempre explícitos, y conviene que este análisis permita superar estas cuestiones. 
Dependiendo del tema del que se trate puede tener mucha significación la confrontación de clases o de bloques sociales, porque no se tiene las mismas motivaciones cuando se manejan recursos abundantes de poder, que cuando se está dependiendo de un salario y unos horarios fijos, o cuando ni siquiera se llega a tener un sueldo más o menos estable. Se pueden hacer muchos tipos de divisiones por razón del poder que se maneja, pero alguna ha de estar presente, porque lo que nos estamos jugando en estos análisis no es tanto una descripción de lo que opinan las personas, sino qué capacidad de intervenir pueden tener, y con qué fuerza. Si lo que queremos es modificar vínculos y relaciones, lo que nos importa es la fuerza con que se pueden influir, y no sólo opiniones generales como si todos fuéramos iguales para decidir. Estamos en un mundo desigual donde las estrategias cuentan para transformar las vidas, pero a partir de reconocer que partimos de situaciones contrapuestas.

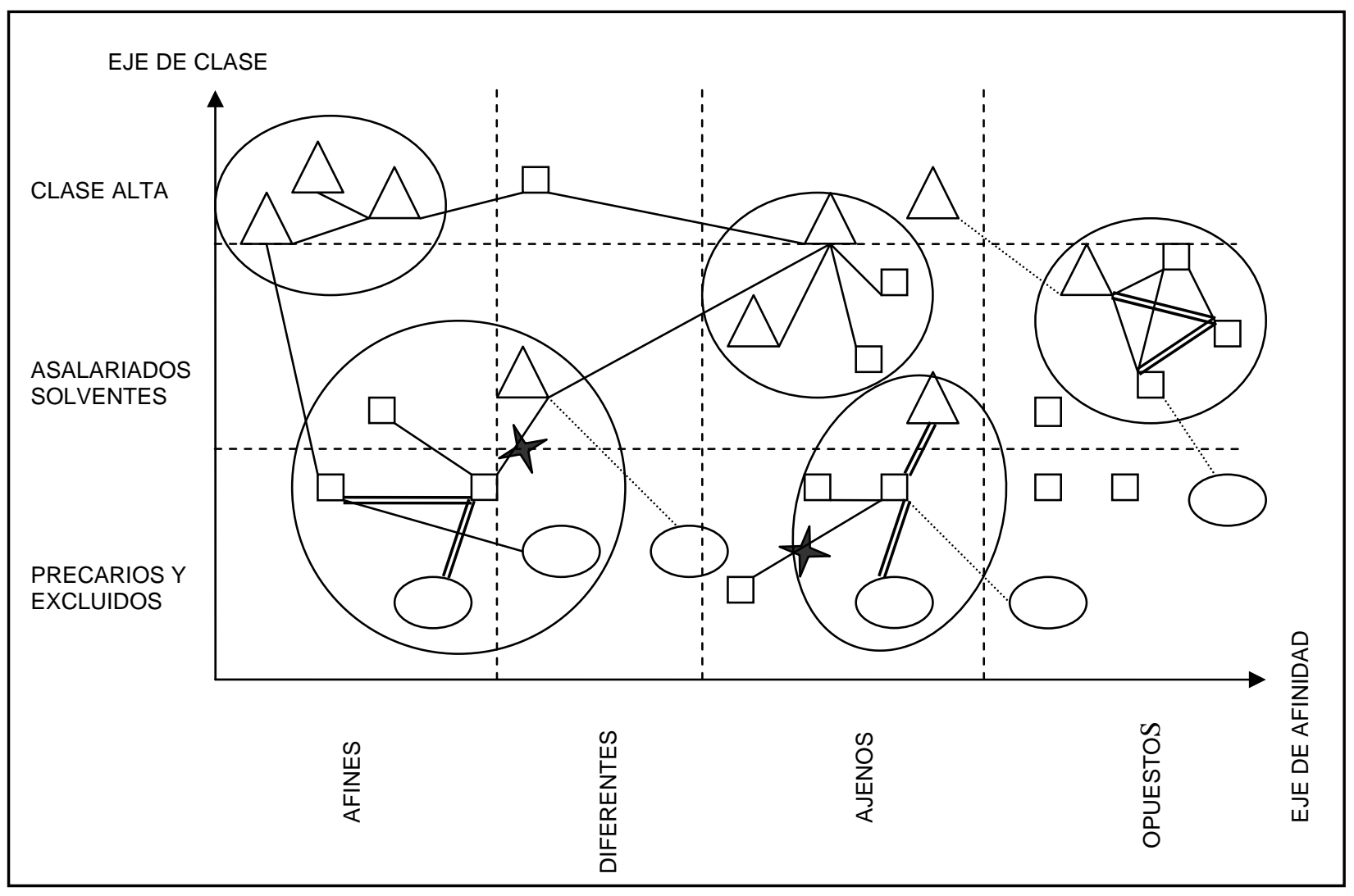

Cuadro 2. La muestra desde el sociograma Fuente: Elaboración propia 
Una muestra ha de representar el universo del que procede; indagando sobre la muestra hemos de poder extrapolar nuestras conclusiones a la población de referencia. Esto es válido tanto para una muestra de una encuesta (distributiva), la de un estudio cualitativo (estructural) o para el caso que nos ocupa, el trabajo desde la perspectiva de las redes sociales (reticular). Si hemos elaborado el sociograma orientado por los ejes y categorías estratégicas que nos parecen más apropiados para el objeto y objetivos de nuestro trabajo, podemos seleccionar una muestra representativa de las relaciones presentes. A partir de este esquema podemos contemplar los vínculos, las posiciones, las subredes de los actores, sus sistemas de relaciones. Porque serán los sistemas de relaciones los que nos van a indicar cómo ha de ser la selección de los elementos de la muestra. Para ello nos apoyamos en un principio general de la teoría de redes: "la estructura de las relaciones sociales afecta al contexto de las relaciones que se pueden dar en la misma"; y en tres dimensiones importantes: “1) el efecto de la posición en la red del actor en su conducta; 2) la identificación de los subgrupos en la estructura de la red y 3) la naturaleza de las relaciones entre los actores, ya sean éstos siempre individuales o colectivos" (Requena, 2003:4-5)

En la realidad de los mapas sociales las posiciones no vienen tan cuadriculadas como se supone por estar construyéndolos sobre unos cuadrantes. Es interesante usar los ejes y los cuadrantes para que no se nos olviden sectores o grupos que no aparecen a primera vista o comentario. Pero lo habitual es que se vayan configurando dentro de un socio-grama algunos "conjuntos de acción" que destacan sobre la maraña de relaciones que se van tejiendo. Volveremos más adelante sobre el tema de los "conjuntos de acción" por la importancia que le damos, pero aquí ya los planteamos para que nos puedan servir de orientación a la hora de enfocar los talleres, entrevistas, grupos de discusión, y otras técnicas y practicas conversacionales que vayamos a usar. Dentro de las múltiples relaciones que se van construyendo entre unos grupos y otros, entre sectores, instituciones, y demás referentes, hay algunos conflictos que muestran barreras y algunas densidades de relaciones que muestran confianzas, hay también zonas en blanco o con pocas relaciones que significan discontinuidades, y hay aglomeración de información sobre grupos en algunos cuadrantes (seguramente los mas afines a los objetivos). 
Dentro de un mapa social o socio-grama podemos, con un mínimo de experiencia, detectar pronto dos o tres "conjuntos de acción" principales, que articulan niveles de poder, ideológicos y de confianzas, en frente de otros. Pueden quedar algunos grupos o sectores sin precisar, algunos sueltos con pocos vínculos o bien otros que aún hemos de precisar su posición por falta de información inicial. A los grupos más afines se les puede convocar a una reunión (taller, grupo de discusión, etcétera.) precisamente porque ya se sabe lo fundamental de sus posturas, y quizás lo que interesan son algunos matices. Y poco más habrá que saber de este conjunto de acción cercano. En cambio nos interesa mucho cómo son las motivaciones de los otros conjuntos de acción, precisamente los que se mueven en las zonas diferentes, antagónicas o ajenas. $Y$ dentro de esos conjuntos aquellas posiciones que pudieran hacer de "puentes" o de "nodos" entre unos sectores y otros. Cuanto más nos alejamos de las posiciones más cercanas a las que nosotros defendemos hay que hilar más fino.

Si estamos hablando de posiciones de instituciones o de grupos formales, es posible que haya escritos que nos den una idea previa, pero también portavoces o personas significativas que hablen en nombre de las reuniones donde ya hayan consensuado sus posiciones. En estos casos se trata de mantener entrevistas o cualquier otra técnica conversacional que muestre su particular forma de razonar y argumentar sus posturas, sean estas cuales sean, sin entrar en juicios. No estamos debatiendo o juzgando sobre la verdad, sino en la fase de documentarnos y escuchar las distintas motivaciones, para precisar la radiografía y el diagnóstico de lo que hay en juego. En muchas de estas entrevistas personales se mezclarán las fantasías con las realidades, pero eso no hace sino confirmarnos desde dónde se posiciona cada cual como colectivo, y en dónde se trata de poner a los otros y las otras desde su punto de vista. 
Cuando se trata de llegar a las opiniones de sectores informales (pandillas de jóvenes, grupos de señoras, peñas de jubilados, y otros grupos de amistad por razones étnicas, deportivas, festivas, etcétera.) lo más conveniente es utilizar técnicas colectivas de entrevistas. La cuestión es que las reuniones de varias personas permitan un efecto de sentirse controlando la situación por el grupo frente a las personas que vienen a preguntar. Si se consigue que una pandilla, por ejemplo, pueda hablar en su terreno y con sus reglas habituales de expresarse, la información producida será más interesante y creativa, que si están a la defensiva como ante un maestro. En los cuadrantes que están más en la base de los conjuntos de acción, o los sectores que están sueltos y que no tienen una organización (muy abundantes) sí es conveniente que no se olviden los criterios de diferenciar por edades, género, condiciones económicas o culturales. Pero la cosa es también que no se repitan tanto los mismos argumentos que estemos perdiendo el tiempo en reiteraciones.

Solemos decir que la muestra esta "saturada" cuando ya se están repitiendo las principales razones centrales en unas y otras posiciones. $\mathrm{Si}$ los ejes de los cuadrantes nos dan entre 9 y 12 posiciones básicas sobre un problema que hemos delimitado previamente, no parece razonable que haya que hacer más técnicas conversacionales que las dichas. Incluso lo más frecuente que nos sucede es que haya 2 ó 3 posiciones dominantes, según cada uno de los "conjuntos de acción", con variables para tales o cuales temas del guión que proponemos. A partir de estas posiciones dominantes por cada tema de interés, lo que nos puede interesar más no es tanto cuánto se repiten en la muestra, sino qué variaciones internas se producen en su formulación. Es decir, intentar llegar a las contradicciones y paradojas que se producen en cada posición y entre posiciones afines tanto como con las diferentes o antagónicas. Estas escuchas nos ponen en las pistas de la complejidad de la materia prima de nuestra labor de análisis y de devolución a la propia comunidad.

Resumiendo: a la vista de qué sistemas de relaciones se dan en cada cuadrícula, podremos ir seleccionando la muestra, teniendo en cuenta en primer lugar a los conjuntos de acción claramente identificables; este será el elemento de homogeneidad más claro. Pero hemos de considerar también las ya mencionadas dimensiones que nos introducen complejidad en los sistemas de relaciones: la posición de los actores (con especial atención a las posiciones de centralidad, densidad o intermediación ${ }^{5}$ ), los subgrupos que no llegan a constituirse en conjuntos de acción y las peculiaridades de los vínculos (contenidos, fuerza, 
reciprocidad, etcétera). Esto no excluye el uso de otras variables de inclusión de heterogeneidad en la muestra, como por ejemplo el género, la generación, el origen cultural, etcétera. Siempre teniendo en cuenta la pertinencia de nuestras elecciones para la estrategia investigadora y transformadora.

En este tipo de análisis de redes no nos interesa tanto cuantificar las reiteraciones de los vínculos, como las paradojas básicas en que se sostienen. Nos interesa más profundizar, ayudados con devoluciones de las propias frases textuales, en "¿por qué dijeron lo que dijeron?", que en entrar a juzgar quién pueda tener razón. Hay razones en plural, y a partir de ahí los vínculos se pueden mover, juntar en confianzas y acciones comunes, o bloquearse en miedos,... Las muestras no son la realidad completa, son como trozos o resúmenes donde intentamos profundizar, ensayar a ver hasta dónde pueden aparecer nuevas posturas que desbloqueen situaciones paralizadas. La creatividad es en estos casos muy importante, y hay que estar atentos a posibles posturas emergentes (muchas veces minoritarias) que abren nuevas vías para hacer avanzar o desbordar situaciones bloqueadas. Ver y oír más allá de las evidencias dominantes nos parece más riguroso e interesante que quedarnos en simplificar lo que más dicen unos y otros.

\section{Conjuntos de acción y estrategias frecuentes}

Hace unos 15 años que venimos comprobando estos diagnósticos de lo que se mueve en la sociedad mediante los "conjuntos de acción". No son delimitados, como los llamados movimientos sociales por sus características clasistas o interclasistas, o de sectores específicos, o por sus temáticas reivindicativas o propositivas, como se suelen hacer simplemente. Se añaden también a dichas características externas el análisis interno de sus vínculos, de sus confianzas y de la densidad de sus relaciones. $Y$ también las relaciones entre los conjuntos en sus procesos, dando cuenta de todas las estrategias en juego en cada contexto completo de una problemática determinada. Por eso (Villasante 1994) preferimos el análisis de conjuntos de acción que las conceptualizaciones de movimientos sociales (tan particularistas de cada movimiento y de cada autor).

Tampoco se trata de análisis tan detallistas necesariamente que sólo tengan en cuenta a cada sujeto de la acción por sí mismo. Se acepta que la relación sujetosujeto, muy "emic" y desde cada estrategia, está en la base de nuestros posicionamientos, pero no se queda ahí. Cada conjunto de acción viene cruzado por enfoques estructurales, ideológicos y convivenciales, no sólo desde dentro de sí, sino también desde las otras posiciones con las que se relaciona. Más importante

\footnotetext{
${ }^{5}$ Los que Granovetter llama “puentes locales” (2000).
} 
que los grupos o sectores que lo componen, son las relaciones o vínculos que le dan dinamismo y tareas, se auto-constituyen por las acciones que ponen en marcha. Esto permite que cambiando el tipo de vínculos establecidos sea posible el cambio de cada conjunto y los cambios más generales en la sociedad. No se trata tanto de cambiar a cada sujeto por si mismo como por los vínculos que mantiene. Cambiando las situaciones relacionales cambia el proceso.

Entre el año 1986 y 89 hicimos estudios de 28 barrios de Madrid que acababan de salir de una década de transformaciones importantes (Villasante et alii, 1989). Sobre todo en algunos barrios pudimos comprobar algo que ya se había podido ver en la historia de los movimientos ciudadanos de Madrid en la transición (Villasante, 1984), es decir, que además de los caracteres estructurales de cada barrio y de las reivindicaciones, contaban tanto o más las formas de organización interna y las redes emocionales de cotidianeidad en que se movían. Con parecidas características externas sin embargo sus resultados eran muy diferentes según las redes cotidianas (horizontales, verticales, desconectadas) que también estaban internamente en el proceso.

Entre el año 1989 y 94 pudimos hacer estudios semejantes en 24 barrios de seis metrópolis de América Latina (Caracas, Bogotá, Lima, Santiago, Buenos Aires, y Salvador de Bahía), buscando diferencias muy acusadas en las condiciones estructurales y culturales (barrios de clases medias, de centros históricos, y también periféricos pobres, unos más antiguos y otros de invasiones más recientes). Lo que tratábamos de encontrar eran las características internas de las redes que los animaban con algunos resultados que dieran algunas semejanzas (Villasante et alii, 1994) Y aunque aparecieron muchos tipos de conjuntos de acción mezclados en cada barrio, también pudimos comprobar que los que más se repetían eran de tres tipos: populistas (verticalistas), gestionistas (elitistas), y ciudadanistas (basistas). Y que las culturas más o menos patriarcales, o pedagógicas, o clientelares, etcétera. que se construyen desde las familias hasta las redes de las instituciones, están en la base de muchas de esas practicas. El papel de las mujeres sobre todo nos da unos índices de cómo se han ido construyendo los vínculos internos, como luego se muestran hacia el exterior tanto los liderazgos como los procesos tanto reivindicativos como clientelares.

Desde el año 1995 hasta la fecha hemos seguido comprobando en numerosas investigaciones, con unas técnicas cada vez más depuradas, como estos conjuntos de acción nos sirven para un primer diagnóstico de los sujetos en presencia, una muestra de las posiciones contrapuestas, una verificación con trabajo de campo, y 
unas devoluciones para dar mayor verosimilitud, y proyección creativa, a las estrategias que se ponen en juego. Desde los equipos de investigación de los cursos que aplican estas metodologías y que tienen presencia en Madrid, Barcelona, Sevilla, Canarias, Ecuador, Perú y Chile, hemos ido pudiendo conocer diferentes variantes y enriqueciendo los diagnósticos y las propuestas. En experiencias de tipo más breve también hemos iniciado en Colombia, México, Argentina, Uruguay y Brasil estos análisis, y esto nos anima a seguir con equipos de estos países profundizando en la utilidad del planteamiento.

Al hacer un socio-grama con los ejes que hemos venido comentando, nos pueden aparecer varios de los conjuntos de acción dichos, y otros grupos o sectores más aislados o sueltos, fuera de los principales conjuntos. En los países referidos nos suelen aparecer sobre todo algunos de estos tres conjuntos que pasamos a referir. Son conjuntos que se mueven en sus relaciones cada cierto tiempo, algunos meses o algún año, por lo que estas radiografías hay que estarlas haciendo cada cierto número de meses. Lo que era de tipo ciudadanista puede pasar a ser gestionista o populista, y también lo que empieza siendo populista se puede convertir en otra tipología, o desde el gestionismo pasarse a otras formas de populismo, etcétera. $Y$ por supuesto puede haber gestionismos con programas mas conservadores o más progresistas, ciudadanismos o basismos no tan progresistas, y populismos con tintes revolucionarios...

Por ejemplo, la comunicación vertical desde iniciativas del poder, a través de grupos y asociaciones intermedias, puede movilizar a las bases sociales, tanto para un proyecto más clientelista como para un proyecto transformador. El riesgo es dejarse a otra parte de las asociaciones por el camino, por no tener esta buena comunicación con el poder establecido. En estas formas de redes no estamos hablando tanto de los contenidos como de los estilos y maneras de generar confianzas y movilizaciones. En Latinoamérica han ocurrido a mitad del siglo anterior varios populismos tanto en las formas verticales de las redes como en los contenidos paternalistas. Pero en este nuevo siglo podemos asistir a fenómenos donde la forma vertical de las redes tiene contenidos manifiestamente de apoyo a un líder pero con contenidos de cambio social fuerte.

Otro tipo de conjunto que a veces ha venido a sustituir al populismo es el que conecta a varios grupos y asociaciones entre sí y con apoyos de los poderes también, pero que no logra llegar a las bases desde el punto de vista de la empatía con ellas: es el que llamamos gestionista. Gestiona bien sobre todo para los diversos colectivos implicados, y guarda cierta participación hasta sus núcleos de 
decisión, pero la gente no se ve incorporada en estos procesos. Puede ser el caso típico de ONGs con apoyos internacionales y locales, pero la movilización y el grado de emoción de los sectores populares quedan lejos de lo que pueda conseguir el populismo o el ciudadanismo. Las relaciones son horizontales entre las instituciones y las asociaciones, pero la inmensa mayoría de la población no se siente metida en el proceso, y en este sentido puede ser considerado elitista su planteamiento. En España suele darse con frecuencia también en las asociaciones locales más veteranas. Un caso extremo es el que aparece en el cuadro siguiente denominado técnico, en el que el actor central, no sólo se ocupa de gestionar las demandas que se le hacen desde el poder, sino que se mantiene aislado e incluso en conflicto con otros actores similares a él, pretendiendo la exclusividad.

El conjunto ciudadanista es más de base y también horizontal, es decir, se vinculan bien los grupos y colectivos entre sí y con los sectores informales no organizados. Es, por ejemplo, el caso típico de una movilización o movimiento popular, sea de tipo más transformador o de reivindicaciones más puntuales. Puede entrar en conflicto con los poderes o en negociación, según las situaciones, pero en cualquier caso siempre tiene una cierta inestabilidad, y ha de cuidar mucho su estructura horizontal de base pues se puede desequilibrar con facilidad. Si no negocia y entra en un conflicto frontal, es algo complicado mantener la tensión durante mucho tiempo, puede quemarse o desgastarse. Si negocia puede desmovilizar a una parte de sus bases, o pasar a ser gestionista o populista. El análisis de redes de estos conjuntos de acción, como se ve, es poco estático, supera por su propio planteamiento las descripciones de redes más habituales de las ciencias sociales.

Estamos usando un concepto de poderes en las relaciones de lo cotidiano, que no hace referencia tanto al poder en sí mismo, como algo establecido, sino como una construcción en proceso. Sin duda estamos en sociedades desiguales, pero unas personas o grupos tienen poder sobre otras más por las relaciones que se establecen que porque las cosas tengan que ser así. O dicho de otro modo, es desde lo cotidiano desde donde se construyen los poderes, en la familia, en las relaciones de trabajo o de educación, en las amistades y las vecindades. Los movimientos de mujeres lo plantearon hace bastante tiempo, y ellas nos enseñaron que el patriarcado es una construcción social. El patriarcado es una forma de vínculos que se impone desde lo emotivo en la familia, y luego se extiende a otras muchas formas de relación en nuestras sociedades (escuelas, asociaciones, estados, etcétera). Nuestro análisis de redes y conjuntos de acción tiene muy presente estas culturas de dominación desde lo micro hasta lo macro. 
Populista

personalista

Gestionista solidarista

Tecnicista aislado

Ciudadanista de base

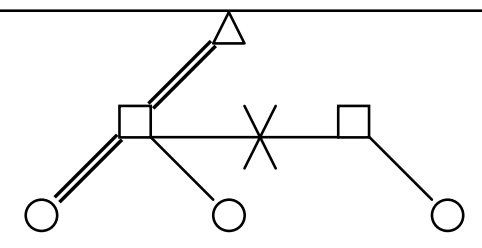

Gestión más movilización. Ruptura con otros grupos
Gestión desde arriba.

Negociación con otros grupos. Asistencia formal con la base
Gestión desde arriba.

Problemas con otros grupos. Asistencia formal con la base
Negociación con el poder. Cooperación con otros grupos. Movilización con la base
ELEMENTOS:

PODER

GRUPOS

BASES

\section{LEYENDA}

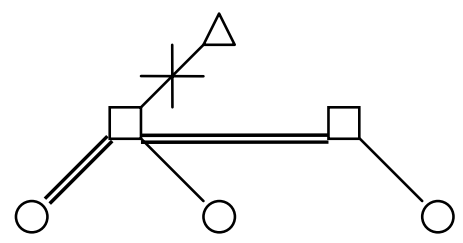

$\bigcirc$

RELACIONES

DENSAS

FORMALES

CONFLICTIVAS

VACÍAS 
Notamos que hay una correlación entre lo que pasa en los ambientes familiares, en las sociedades civiles locales, y en los estados. Y que los procesos de cambio si sólo se producen a nivel local, y no consiguen cambiar las formas societales y estatales tienen muy difícil consolidarse, de la misma forma que si solo hay un cambio del poder por arriba, pero las formas vinculares siguen siendo las mismas por abajo, tal poder acabará por bloquearse y degenerar. En este sentido si los estudios de redes incorporan los factores de "tiempo" y de "poder" tal como lo estamos proponiendo con los conjuntos de acción, se pueden convertir en un factor de análisis y de intervención muy valiosos para las ciencias sociales. Todo conjunto de acción tiene sus estrategias más o menos explícitas, y en todo caso hemos de saber cuales pueden ser para movernos en el juego de poderes que representan.

\section{Reversión o desborde frente a dilemas: desbloqueo de discursos}

Un nuevo paso en el que hemos trabajado estos últimos años es la cuestión de cómo saltar de unos conjuntos de acción a otros, cómo enfrentar los conflictos internos y externos en cada situación. Los poderes de lo cotidiano no sólo son "poderes sobre", sino también "poderes para". Las relaciones no son sólo de dominación sino de construcción. No se trata de "tomar" los poderes sino de construirlos o reconstruirlos, y para eso a veces hay conflictos muy fuertes. En las relaciones de las redes hay situaciones muy paradójicas, pues las dialécticas complejas están dentro de estas mallas de vínculos. De poco sirve algunas cuestiones de tipo dicotómico, dilemas simples entre esto y aquello, como si sólo pudiéramos jugar entre dos posturas. A veces la dialéctica se ha entendido en estos simplismos y así nos ha ido... o "reforma" o "revolución", o progresistas o conservadores, etcétera. Nos parece que hay un cierto maniqueísmo detrás de muchas de estas posturas, a veces incluso con pretensiones transformadoras.

Hemos aprendido precisamente con los movimientos sociales a ser más pragmáticos, y no tan rígidos o "principistas", porque a diferencia de algunos colectivos más minoritarios o elitistas, los movimientos sí que han de dar algunos resultados a sus implicados, si quieren mantener el interés y la motivación de los participantes de base. Esto no quiere decir que haya que caer en el otro polo de la dicotomía, es decir, que por ser pragmáticos se ha de aceptar cualquier propuesta. Sí quiere decir que se ha de salir de la dicotomía y conseguir al tiempo la reforma y la revolución. Se trata de entender que no son conceptos excluyentes en las prácticas cotidianas de los conjuntos de acción. El rigor no estará por tanto centrado en mantener unos principios teóricos incuestionables, sino un proceso participativo y creativo que incremente todo lo que nos conduzca al cambio transformador, pero al ritmo de la gente. 
El concepto de "desborde popular" está vinculado a las redes de lo cotidiano. En algunos momentos, muchas veces inesperados, ocurren movilizaciones que nos sorprende a todos, incluidos a los propios participantes, porque algún factor de lo cotidiano acaba por desbordar una situación que acumulaba ya bastantes contradicciones. No se trata sólo de que hay un programa más o menos radical, sino de que la gente se sienta implicada o no en el proceso. Puede ser que se trate de conseguir reformas, pero si se hace por movimientos de organización popular, y se va creando una conciencia de poder alternativo, es que desde lo interno se está fraguando un cambio más estructural; y también pude ser que apostando por unas transformaciones más radicales el proceso se quede en algunas conquistas de reformas, que den durante un tiempo posibilidades para una mejor organización social. En los movimientos que han perdurado con cierta historia se suelen suceder momentos con todas estas complejidades y más.

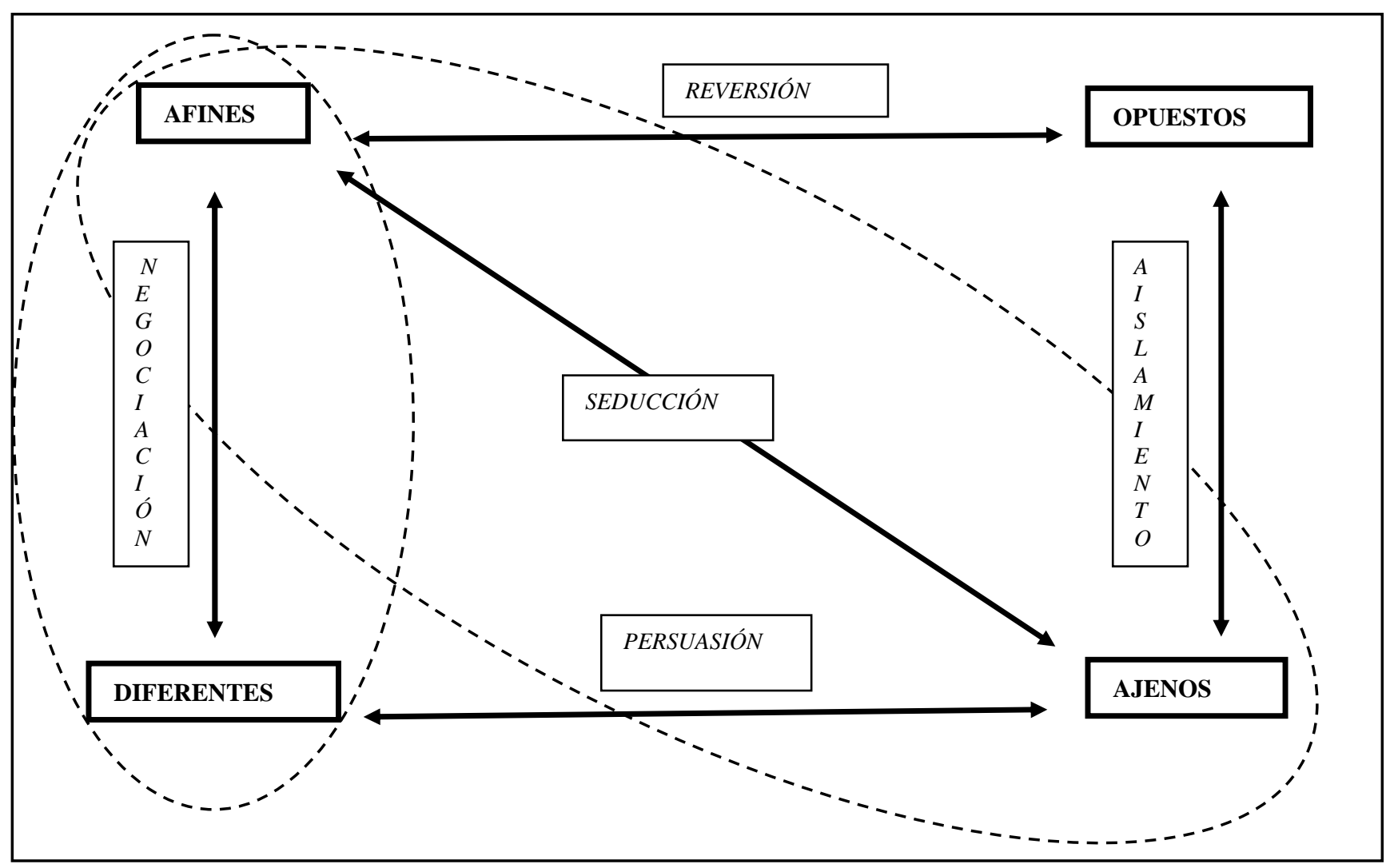

Cuadro 4. Posiciones de afinidad de los actores y construcción de posibles conjuntos de acción Fuente: Hernández, Martín y Villasante (2000:38) 
Estos desbordes también los podemos caracterizar con el concepto de reversión (Ibáñez, 1994: 66-79) en la medida en que desde este punto de vista también quedarían superadas las dicotomías y las dialécticas simples habituales en muchos cientistas sociales. Frente a una posición con dos polos, puede uno quedarse en posiciones intermedias, pero no se sale de la línea de confrontación. Lo que se plantea en este caso es salirse a otro plano, por ejemplo, entre "lo uno" y "lo otro", optar por ampliar las posibilidades de elección a "ni lo uno, ni lo otro", no aceptar la pregunta de la disputa y reformular la polémica de otra manera, posiblemente más profunda. O bien aceptar que "lo uno y lo otro" no son incompatibles, encontrar la contradicción en la pregunta misma, y revertirla mostrando por ejemplo su hipocresía. Ir tan a fondo con una alternativa creativa que desbordemos por los hechos a los que han formulado la cuestión de forma "tramposa".

Si tratamos de emplear esta lógica en relación con los conjuntos de acción que suelen aparecer en nuestros sociogramas, podemos intentar desbloquear las situaciones más cristalizadas y enquistadas que aparecen en el territorio. Cuando hay un enfrentamiento entre dos conjuntos de acción, por ejemplo entre el de los más afines a los objetivos del proyecto, y el de los opuestos o antagónicos, es muy posible que tal lucha acabe por bloquear el desarrollo y la superación del problema. Hay que prestar atención a las otras redes que aunque no parezcan estar en juego son las que pueden decidir, o al menos desbloquear la situación. Seguramente hay otras redes $u$ otro conjunto que siendo cercano es también diferente en los estilos respecto al conjunto afín, y desde luego hay numerosos grupos y sectores que están ajenos en principio al tema, y que sólo van a entrar en función de las propuestas que se vayan formulando, o seguirán al margen. En situaciones como estas el enfrentamiento directo con los antagonistas sólo suele llevar a insultarse y descalificarse, pues no es creíble que se les vaya a convencer o concienciar solo con discursos.

Planteado este modelo de situación, con estos términos, lo que se propone en el cuadro 4 es la posibilidad de iniciar, mediante la aplicación de un proceso dialógico, la construcción de más amplios conjuntos de acción, la construcción de nuevas redes que puedan afrontar el reto de otras propuestas transformadoras. De ahí que, partiendo de este nosotros que suponen los actores más afines, pasar a una negociación con los conjuntos de acción diferentes; unos y otros pueden crear un espacio donde la toma de decisiones sea de carácter más interna a los actores implicados. Podemos estar hablando de redes efectivas que tienen ya experiencia de vínculos comunes. 
Otro caso es el de la ampliación del posible conjunto de acción a los ajenos, los que se mueven en otras lógicas, en otros espacios de menos afinidad, más alejados. En este caso la estrategia pasaría más por la seducción o la persuasión: "la seducción nunca es del orden de la naturaleza, sino del artificio -nunca del orden de la energía, sino del signo y del ritual. [...]¿Por qué un desafío exige respuesta? ¿Qué hay de más seductor que el desafío? Desafío o seducción, es siempre enloquecer al otro, pero de un vértigo respectivo, locos de la ausencia vertiginosa que los reúne y de una absorción respectiva" (Baudrillard 1998:79). Enloquecer al otro y enloquecer con el otro, es la posibilidad de establecer vínculos sobre la base de un plan de acción realmente seductor, que desborde las lógicas más trilladas: "lo llevamos a cabo porque cuando nos lo propusimos no sabíamos que era imposible", era una frase recogida en una experiencia comunitaria. Es una magnífica expresión de a qué nos referimos con lo de seducción, el desbordar lo razonable.

Por último, las posibilidades de agrupar a todos los actores llegan al límite sobre todo cuando se ponen en prácticas las propuestas. Los acuerdos universales se desmoronan en lo concreto: ¿quién no va a desear el bienestar, la salud, el desarrollo sustentable... para todos?. Los desacuerdos comienzan al poner estas proclamas en acciones, cuando la salud o el desarrollo sostenible pasan por modificar procesos productivos insalubres o insustentables, cuando los recursos escasos hay que distribuirlos, cuando el balance de problemas afecta a unos o a otros sectores de una comunidad. Entonces es cuando aparecen propuestas antagónicas y sobre los que hay que decidir también desde la lógica de redes. La reversión y el aislamiento son estrategias para reducir las posibilidades de los sectores con intereses antagónicos y para situar a los conjuntos de acción que las sostienen en la posición más desfavorable para construir sus propuestas.

Estas lógicas y estas estrategias las hemos contemplado, aprendido y ensayado en situaciones y con actores (desde personas significadas en sus comunidades hasta movimientos sociales) que las manejaban sin hablar de redes sociales, hasta momentos muy recientes, en los que este y otros términos semejantes aparecen en el lenguaje común. La operativización, la comprensión y explicación de estas estrategias, de estas lógicas, es tarea de científicos, pero no sólo... Desde posiciones críticas y reflexivas no podemos olvidar el "para qué" y el "para quién" de estas explicaciones, es decir, la tesis marxiana de que las cosas se hacen para transformar, no sólo para comprender mejor. 


\section{Bibliografía}

Baudrillard, J. (1998). De la seducción. Madrid: Cátedra.

Dabas, E.N. (1993). Red de redes. Buenos Aires: Paidós.

Granovetter, M. (2000). "La fuerza de los vínculos débiles", en Política y Sociedad, no 33: 41-56. Madrid: Facultad de CC. Políticas y Sociología de la UCM.

Hernández, Mạ D.; Martín, P y Villasante, T. (2002). “Estilos y coherencias en las metodologías participativas", en Villasante, T. y Garrido, F.J. Metodologías y presupuestos participativos. Construyendo ciudadanía / 3. Madrid: CIMAS-IEPALA. (pp. 17-42).

I báñez, J. (1994). Por una sociología de la vida cotidiana. Madrid: Siglo XXI.

Lomnitz, L. (1977). Networks and marginality. Nueva York: Academic Press.

Martí, J. (2000). “La Investigación-acción participativa: estructura y fases", en Villasante, Montañés y Martí, La investigación social participativa. Construyendo ciudadanía / 1. Barcelona, El Viejo Topo (pp. 73-118).

Martín, P. (1999). "El sociograma como instrumento que desvela la complejidad", en Empiria, no 2 (pp. 129-151).

Martín, P. (2001). "Mapas sociales: método y ejemplos prácticos", en VILLASANTE, Montañés y Martín, Prácticas locales de creatividad social. Construyendo ciudadanía / 2. Barcelona: El Viejo Topo (pp. 91-113).

Montañés, M. (2003). “El diseño del proyecto de investigación/planificación social participada", en Encina, J; Avila, M. Fernández, M. y Rosa, M. (coord.) Praxis participativas desde el medio rural. Madrid: IEPALA-CIMAS (pp.117-132).

Montañés, M. (2005). “Las muestras", en Martí, J.; Pascual, J. y Rebollo, O. (coord.) Participación y desarrollo comunitario en medio urbano. Construyendo Ciudadanía / 7. Madrid, CIMAS-IEPALA (pp. 293-307).

Pichon Riviere, E. (1991). Teoría del vínculo. Buenos Aires: Nueva Visión.

Requena Santos, F. (2003). Análisis de redes sociales. Orígenes, teorías y aplicaciones. Madrid, CIS - Colección Monografías, 198.

Villasante, T. (2006). Desbordes Creativos. Estilos y estrategias para la transformación social. Madrid: La Catarata.

Villasante, T. (2002). Sujetos en movimiento. Construyendo Ciudadanía / 4. Montevideo: NORDAM-COMUNIDAD.

Villasante, T. (1994) (coord.). Las ciudades hablan. Caracas (Venezuela): Editorial Nueva Sociedad.

Villasante, T. (1984). Comunidades locales. Madrid: IEAL.

Villasante, T. et alii (1989). Retrato de chabolista con piso. Madrid, IVIMA-SGVALFOZ

VV.AA. (2000). Política y Sociedad, no 33. Fac. de CC. Políticas y Sociología - UCM. Número monográfico de Análisis de redes sociales 